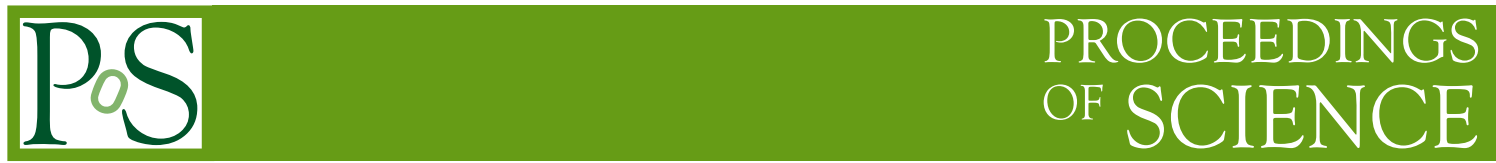

\title{
Spin determination of single-produced resonances at the LHC
}

\section{Nhan Viet Tran*}

Rowland Dept. of Phys. and Astron.-Johns Hopkins University

E-mail: nhan.viet.tranecern.ch

We study the production of a single resonance at the LHC and its decay into a pair of $Z$ bosons. We demonstrate how full reconstruction of the final states allows us to determine the spin and parity of the resonance and restricts its coupling to vector gauge bosons. Full angular analysis is illustrated with the simulation of the production and decay chain including all spin correlations and the most general couplings of spin-zero, -one, and -two resonances to Standard Model matter and gauge fields. We note implications for analysis of a resonance decaying to other final states.

35th International Conference of High Energy Physics

July 22-28, 2010

Paris, France

\footnotetext{
* Speaker.
} 
Physics beyond the Standard Model (SM), to be probed at the LHC will manifest itself through observations of new particles. To fully understand the nature of any New Physics; the spin, CP, and couplings to the SM fields of a new particle must be determined as accurately as possible. We study the decay of new particles at the Large Hadron Collider (LHC) via quark-antiquark or gluongluon partonic production processes into a pair of $Z$ bosons [1]. The $Z Z$ decay to four leptons final state is optimal for such studies because the leptons are reconstructed fully and accurately, more information is contained a four-body final state, and in many cases, the decay of a new particle to a pair of $Z$ bosons may be a large, or possibly dominant, contribution. As an example, possible new particles which we may expect to find include the spin-zero SM Higgs boson [2] whose decay to $Z Z$ is dominant above the threshold, $2 m_{Z}$, twice the mass of the $Z$ boson. However, there are many other possibilities such as new gauge bosons [3] or a spin-two graviton which appears in extra-dimensional models [ 4 . 5]. In this study, we take a model-independent approach so as not to bias ourselves to any particular beyond the SM scenario. We consider general couplings of any spin-zero, spin-one, or spin-two particle to the SM fields. By studying the angular distribution of

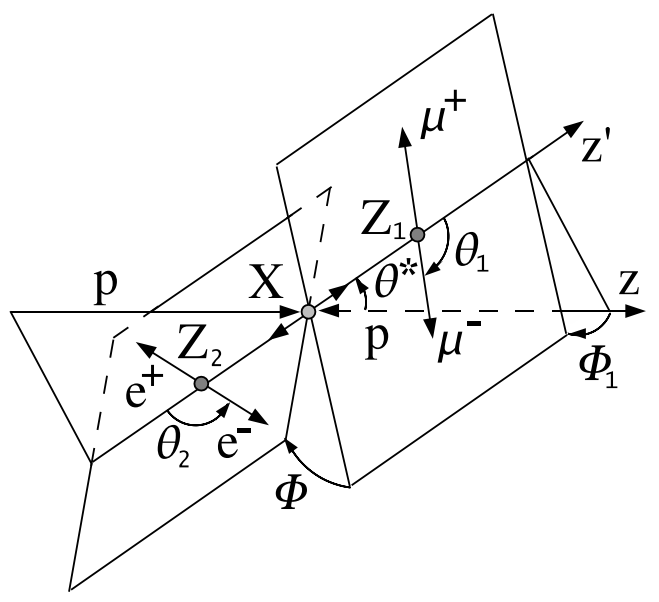

Figure 1: Illustration of an exotic $X$ particle production and decay in pp collision $g g$ or $q \bar{q} \rightarrow X \rightarrow Z Z \rightarrow 4 l^{ \pm}$. Six angles fully characterize orientation of the decay chain: $\theta^{*}$ and $\Phi^{*}$ of the first $Z$ boson in the $X$ rest frame, two azimuthal angles $\Phi$ and $\Phi_{1}$ between the three planes defined in the $X$ rest frame, and two $Z$-boson helicity angles $\theta_{1}$ and $\theta_{2}$ defined in the corresponding $Z$ rest frames. The offset of angle $\Phi^{*}$ is arbitrarily defined and therefore this angle is not shown.

the decay products of the $Z Z \rightarrow 4 l$ system, we can determine the spin and $\mathrm{CP}$ properties of a given resonance. The system is maximally described by five angles. The angles are defined and illustrated in Fig. 1. The analysis is performed using the per-event multivariate likelihood method to extract the maximal amount of information from the system. Such analyses in hadron collider physics have been performed in determinations of the top quark mass and have been widely used in many $B$ physics measurements [9]. The goal of this study is to apply these methods to the determination of a spin of a resonance produced in hadron collisions. Many past works have been dedicated to this topic [6, 7, 8]; our goal was to extract new particle properties in a model-independent way using the maximal amount of information of the system including all five angles and the multivariate 
likelihood method. For further detailed discussion of this study, refer to [1].

To determine the angular distributions of a new particle, we start by constructing the most general amplitudes consistent with Lorentz invariance and Bose-symmetry, as well as gauge-invariance with respect to unbroken subgroups of $S U(3) \times S U(2)_{L} \times U(1)_{R}$ of the SM. From these general amplitudes, we calculate the helicity amplitudes for the production and decay processes. Helicity amplitudes are important because those amplitudes parameterize angular distributions and, hence, can be directly extracted from data. By knowing how these amplitudes are expressed through effective couplings introduced in the general amplitudes, we can constrain those couplings through measurements of angular distributions. Recall, the angular system was described in Fig. 1. Distributions of the five angles allow one to determine the spin of an $X$ boson and measure contributions of the different helicity amplitudes in both production and decay. We use the helicity formalism as described in Refs. [10, 11]. For examples of the angular distributions, see Fig. 2, which has the projections of the five angles for various example scenarios of spin-zero, -one, and -two particles. The smooth lines are the analytically derived angular distributions while the points are generated by a dedicated Monte Carlo written for this study [12].

To determine the sensitivity of our analysis, we use the MC simulation to generate both signal and background events, and apply a multivariate fitting technique to extract as much information about the produced resonances as possible. Because our analysis is general and is not based on any particular model of BSM physics, it is not possible to accurately predict how many new particles are produced for a given luminosity. Instead, we find it more reasonable to assume a certain number of signal and background events, reconstructed by an LHC experiment. As a particular example, we select 30 signal events which corresponds to approximately $5 \mathrm{fb}^{-1}$ of data for the SM Higgs. With this relatively small sample of signal events, for example, we can distinguish between a SM Higgs and a new pseudoscalar particle hypothesis with $4.1 \sigma$. More complete results are given in [1] for many different signal scenarios. By comparing various possibilities and maximizing all available information using all five angles and the multivariate likelihood method, we find that, at the time of discovery of any new resonance, we will already be able to make a statement about the spin and $\mathrm{CP}$ of the new particle.

The author wishes to acknowledge collaborators Yanyan Gao, Andrei Gritsan, Zijin Guo, Kirill Melnikov, and Markus Schulze.

\section{References}

[1] Y.Y. Gao, A. V. Gritsan, Z.J. Guo, K. Melnikov, M. Schulze, N. V. Tran, Phys. Rev. D 81, 075022 (2010).

[2] For review of Higgs studies see G. Bernardi, M. Carena, and T. Junk, page 414 of Particle Data Group, Phys. Lett. B 667, 1 (2008).

[3] W. Y. Keung, I. Low, and J. Shu, Phys. Rev. Lett. 101, 091802 (2008).

[4] B. C. Allanach, K. Odagiri, M. A. Parker, and B. R. Webber, JHEP 0009, 019 (2000).

[5] K. Agashe, H. Davoudiasl, G. Perez, and A. Soni, Phys. Rev. D 76, 036006 (2007).

[6] C.P. Buszello, I. Fleck, P. Marquard, and J.J. van der Bij, Eur. Phys. J. C32, 209 (2004).

[7] S.Y. Choi, D. J. Miller, M.M. Muhlleitner, and P. M. Zerwas, Phys. Lett. B553, 61 (2003). 

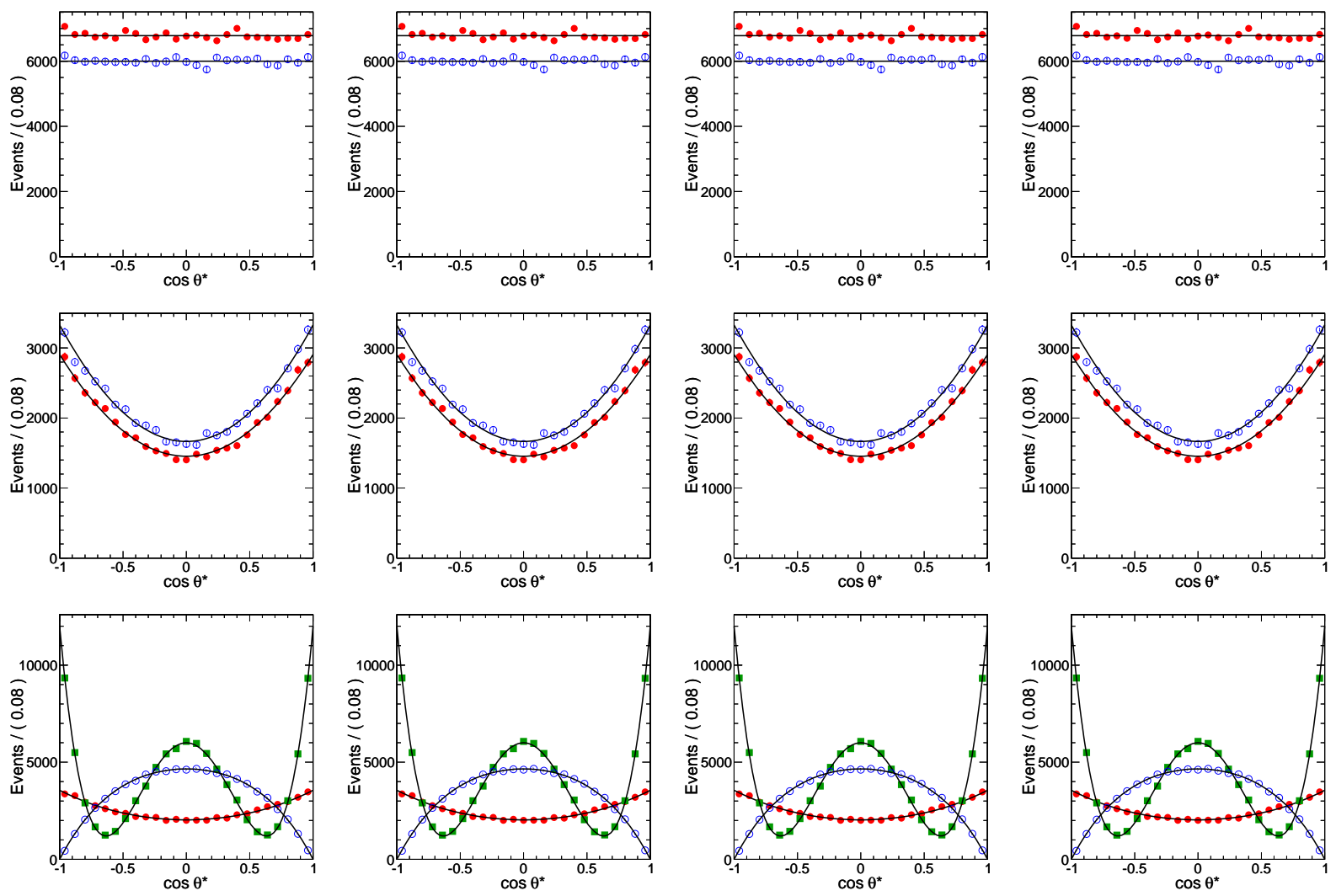

Figure 2: Distribution of the $\cos \theta^{*}$ (left), $\Phi_{1}$ (second from the left), $\cos \theta_{1}$ and $\cos \theta_{2}$ (second from the right), and $\Phi$ (right) generated for $m_{X}=250 \mathrm{GeV}$ with the Monte Carlo program discussed in the text (unweighted events shown as points with error bars) and projections of the angular distributions (smooth lines). The three sets of plots from top to bottom show the models for spin-zero $0^{+}$and $0^{-}$(top), spin-one $1^{+}$and $1^{-}$(second row from top), and spin-two $2_{m}^{+}, 2_{L}^{+}$, and $2^{-}$(third row from top). The $J^{+}$distributions are shown with solid red points and $J^{-}$distributions are shown with open blue points, while the $2_{m}^{+}$and $2_{L}^{+}$ are shown with red circles and green squares, respectively.

[8] A. Soni and R. M. Xu, Phys. Rev. D 48, 5259 (1993).

[9] V. Barger, Y. Gao, and W.-Y. Keung, "T-Anomaly Induced LHC Signals," Phys. Lett. B655, 228 (2007).

[10] T. L. Trueman, Phys. Rev. D 18, 3423 (1978).

[11] J. R. Della'Aquila and C. A. Nelson, Phys. Rev. D 33, 80 (1986).

[12] www.pha.jhu.edu/spin 